Radial and Nonradial Pulsations as Probes of Stellar Physics

ASP Conference Series, Vol. 259, 2002

C. Aerts, T.R. Bedding, \& J. Christensen-Dalsgaard, eds.

\title{
Multiperiodicity in the Delta Scuti Star HR 4047
}

\author{
A. Rolland, E. Rodríguez, V. Costa, I. Olivares, J.M. García-Pelayo, \\ P. López de Coca \\ Instituto de Astrofísica de Andalucía, Granada, Spain \\ M.A. Hobart \\ Facultad de Física, Universidad Veracruzana, Xalapa, Veracruz, M exico
}

HR 4047 (HD 89343, EN UMa, V = 5m92, A7Vn) was found to be variable (Plume \& Percy, 1988) with an amplitude of hundredths of a magnitude. Since then, this star has been observed by HIPPARCOS. Koen (2001), analysing those data, obtained two frequencies $6.43185 \mathrm{c} \mathrm{d}^{-1}$ and $4.31308 \mathrm{c} \mathrm{d}^{-1}$.

Our observations were made at the Observatorio de Sierra Nevada in Spain in 1997 and 1998 with the telescope of $0.9 \mathrm{~m}$. The comparisons stars for 1997 were $\mathrm{C} 1=\mathrm{HD} 88983(\mathrm{~V}=5 \mathrm{~m} \cdot 77, \mathrm{~A} 8 \mathrm{III})$ and $\mathrm{C} 2=\mathrm{HD} 88815\left(\mathrm{~V}=6{ }^{\mathrm{m}} \cdot 42, \mathrm{~F} 2 \mathrm{~V}\right)$, while for 1998 we add the check star $\mathrm{C} 3=\mathrm{HD} 90838(\mathrm{~V}=7.30, \mathrm{~F} 2)$. The photometric data obtained were analyzed using the Fourier Transform method in the Strömgren $v$ filter. The periodogram of the $v$ band data is shown in Fig. 1. The periodogram presents six clear frequencies and after prewhitening these there is still more power, but the frequencies found are not consistent between the different bands. Applying these frequencies to the data of the 1997 and 1998 separately, we obtain the results shown in Table 1. Note that the amplitudes have changed from one year to another. The results of 1998 agree with the two frequencies found by Koen (2001). Fig. 1 shows the light curves of the observed data for both campaigns in the $v$ filter. The points represent the observations while the solid lines represent the fit for the frequencies listed in Table 1. Further observations are needed to obtain a more detailed pulsational behaviour of this star.

Table 1. Results of the Fourier analysis of the $v$ data.

\begin{tabular}{ccc}
\hline & 1997 & 1998 \\
\hline$f\left(\mathrm{c} \mathrm{d}^{-1}\right)$ & Amp (mag) & Amp (mag) \\
\hline 4.3133 & 0.0260 & 0.0288 \\
6.4317 & 0.0213 & 0.0330 \\
7.1469 & 0.0130 & 0.0123 \\
5.8458 & 0.0096 & 0.0058 \\
3.5804 & 0.0071 & 0.0048 \\
4.9885 & 0.0057 & 0.0031 \\
\hline
\end{tabular}



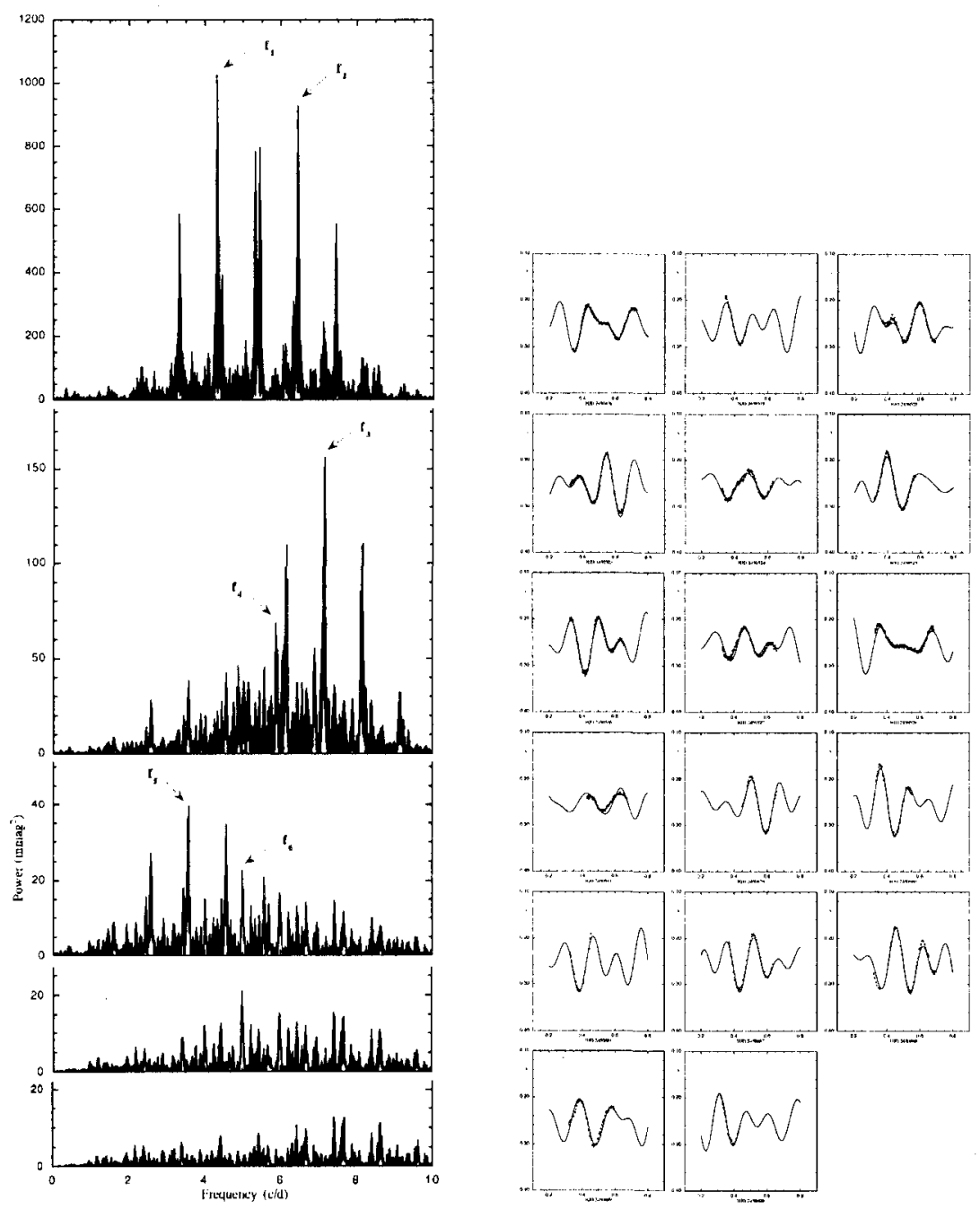

Figure 1. Left: periodogram of the $v$ band for 1997-98. Right: light variations and synthetic light curves.

Acknowledgments. This research was supported by the Junta de Andalucía and the Dirección General de Investigación (MCYT) under project AYA 2000-1580.

\section{References}

Koen. C. 2001, MNRAS 321, 44

Plume, R. \& Percy, J.R. 1988, IBVS, 3225 